\title{
Sleeve lobectomy compared with pneumonectomy for operable centrally located non-small cell lung cancer: a meta-analysis
}

\author{
Zhengjun Li ${ }^{1}$, Wei Chen ${ }^{2}$, Mozhu Xia ${ }^{3}$, Hongxu Liu ${ }^{2}$, Yongyu Liu ${ }^{1}$, Ilhan Inci ${ }^{4}$, Fabio Davoli ${ }^{5}$, \\ Ryuichi Waseda ${ }^{6}$, Pier Luigi Filosso ${ }^{7}$, Abby White ${ }^{8}$ \\ ${ }^{1}$ Department of Thoracic Surgery, Shenyang Chest Hospital, Shenyang 110044, China; ${ }^{2}$ Department of Thoracic Surgery, Cancer Hospital of China \\ Medical University/Liaoning Cancer Hospital, Shenyang 110042, China; ${ }^{3}$ Department of Thoracic Surgery, The First Affiliated Hospital, China \\ Medical University, Shenyang 110001, China; ${ }^{4}$ Department of Thoracic Surgery, University Hospital, University of Zurich, Zurich, Switzerland; \\ ${ }^{5}$ Department of Thoracic Surgery, AUSL Romagna, S. Maria delle Croci Teaching Hospital, Ravenna, Italy; ${ }^{6}$ Department of General Thoracic, \\ Breast, and Pediatric Surgery, Fukuoka University, Fukuoka, Japan; ${ }^{7}$ Unit of Thoracic Surgery, Department of Surgical Sciences, University of \\ Torino, Torino, Italy; ${ }^{8}$ Division of Thoracic Surgery, Department of Surgery, Brigham and Women's Hospital, Boston, MA, USA \\ Contributions: (I) Conception and design: Z Li; (II) Administrative support: H Liu, Y Liu; (III) Provision of study materials or patients: Z Li, W Chen, \\ M Xia; (IV) Collection and assembly of data: Z Li, W Chen, M Xia; (V) Data analysis and interpretation: Z Li, W Chen, M Xia; (VI) Manuscript \\ writing: All authors; (VII) Final approval of manuscript: All authors. \\ Correspondence to: Hongxu Liu. Department of Thoracic Surgery, Cancer Hospital of China Medical University/Liaoning Cancer Hospital, Shenyang \\ 110042, China. Email: hongxuliu@qq.com; Yongyu Liu. Department of Thoracic Surgery, Shenyang Chest Hospital, Shenyang 110044, China. \\ Email: freeman-2006@163.com.
}

Background: The purpose of this meta-analysis was to evaluate evidence comparing sleeve lobectomy (SL) and pneumonectomy (PN) in the treatment of non-small cell lung cancer (NSCLC).

Methods: The English literature search was undertaken in January 2018 and included studies dating back to 1996. Comparative studies were identified, evaluating survival, local recurrence, and distant recurrence rates, operative mortality, 30-day mortality, as well as complications. A pooled odds ratio (OR) and 95\% confidence intervals ( $95 \% \mathrm{CI}$ ) were calculated with either the random or fixed-effect model.

Results: A total of 27 studies were identified, with publication dates between 1996 and 2018. These 27 studies included a total of 14,194 patients: 4,145 treated with SL and 10,049 treated with PN. The overall survival was significantly higher in the SL group compared to the PN one at 1, 3, 5 years. In patients with N0 and N1 disease, 5-year survival rates following SL exceeded those following PN. There was no statistically significant difference in the 3-, 5-year overall survival of N2 patients, according to the extent of surgery. The PN group had a higher rate of operative mortality, 30-day mortality and distant recurrence incidence. However, no statistical difference in complications and local recurrence between SL and PN were observed.

Conclusions: SL is an effective treatment option for hilar NSCLC with improved long-term survival compared to $\mathrm{PN}$, with no increase of recurrence rate or postoperative complications. Furthermore, N2 disease is an important factor related to survival, and lymph node downstaging is a favorable prognostic factor.

Keywords: Sleeve lobectomy (SL); pneumonectomy (PN); lung cancer; meta-analysis

Submitted Sep 10, 2019. Accepted for publication Oct 12, 2019.

doi: $10.21037 /$ tlcr.2019.10.11

View this article at: http://dx.doi.org/10.21037/tlcr.2019.10.11 


\section{Introduction}

Lung cancer is the most common cancer in the world. Surgery remains the standard of care for localized disease, even when the location or extent of the disease needs pneumonectomy $(\mathrm{PN})$ to be radically treated. The treatment of centrally located tumors has always been controversial. Bronchial sleeve resection was introduced in 1947 (1) by Thomas as a means to preserve functional lung parenchyma in patients with compromised pulmonary function; the first sleeve lobectomy (SL) report dates $1954(1,2)$ by Allison and Coll. SL was considered as an alternative to $\mathrm{PN}$ since then several institutions worldwide (2-7) proposed SL in alternative to PN. Recent studies $(8,9)$ have shown that SL compared to PN can improve lung cancer patients' quality of life also prolonging long-term survival. Most surgeons agree that SL has to be performed with technical precision in order to accomplish surgery complete tumor resection without increasing complications. Some recent studies suggested that sleeve resection should be used routinely in the management of patients with anatomically appropriate hilar tumors, regardless of preoperative lung function (10-13). One criticism to SL over PN is their potential risk for local recurrence. These reports suggest that local recurrence following SL was comparable, and long-term survival was similar to or better than after PN. Unfortunately, most of the reports show that tumor' TNM stage distribution is very different between the two surgical approaches, and comparisons of the survival and local recurrence rates of comparison for the 2 different approaches over an appropriate stage stratification is not easy to perform.

The primary purpose of our research was to compare SL and PN's outcome, focusing on (I) survival rates, (II) local and distant recurrence rates, (III) operative mortality, (IV) 30-day mortality, and (V) type and rate of complications of these procedures, as well as to evaluate whether SL can be accepted as a favorable alternative procedure to $\mathrm{PN}$ for patients with non-small cell lung cancer (NSCLC).

\section{Methods}

\section{Study selection}

Electronic searches were performed of the MEDLINE, EMBASE databases and Cochrane Controlled Trial Register (CENTRAL) until January 2018. We use the following Mesh search headings to search: (sleeve lobectomy), (pneumonectomy), (centrally located lung cancer or lung carcinoma), (comparative study) and [randomized controlled trials (RCTs)] in English literature (Figure 1).

\section{Data extraction and quality assessment}

Three independent researchers followed the standard procedures for data extraction. The recorded data included: (I) the number of patients, (II) the 1-, 3-, 5-year overall survival, (III) the local and distant recurrence rates, (IV) the operative mortality, (V) the 30-day mortality, and (VI) the complications rates. The quality of all selected documents was ranked following the score of the non-randomized controlled clinical trial quality evaluation standard.

\section{Study selection criteria}

Inclusion criteria for this study were as follows: (I) absence of extrapulmonary metastasis; (II) lung function was appropriate for PN; (III) no prior or concurrent other neoplasms; (IV) the patients should be suitable for treatment with either SL or PN, and (V) they should have similar baseline demographics.

\section{Exclusion criteria}

The following publication criteria were excluded: abstracts, letters, editorials and expert opinions, reviews without original data, case reports and studies lacking control groups. Studies evaluating unresectable lung cancer or recurrence after lobectomy and those without reported outcomes of interest were excluded.

\section{Surgical technique}

The sleeve resection includes the resection of one lobe and the end-to-end bronchial anastomosis. The procedure for SL is the same as for standard lobectomy until the bronchus is isolated. The bronchus needs a single sharp incision divides, and the incision should avoid ragged edges. It should be noted that beyond the proposed line of the transaction, it is taken not to revascularize the bronchus. Several anastomosis techniques are described, mainly depending on the surgeon's expertise and preference; even if any of them have to respect the basic surgical and oncological principles: (I) achieve tumor-free bronchial margins at the frozen section; (II) perform a tension-free anastomosis; (III) try to avoid bronchial mismatch and (IV) proceed with an en-bloc resection. 


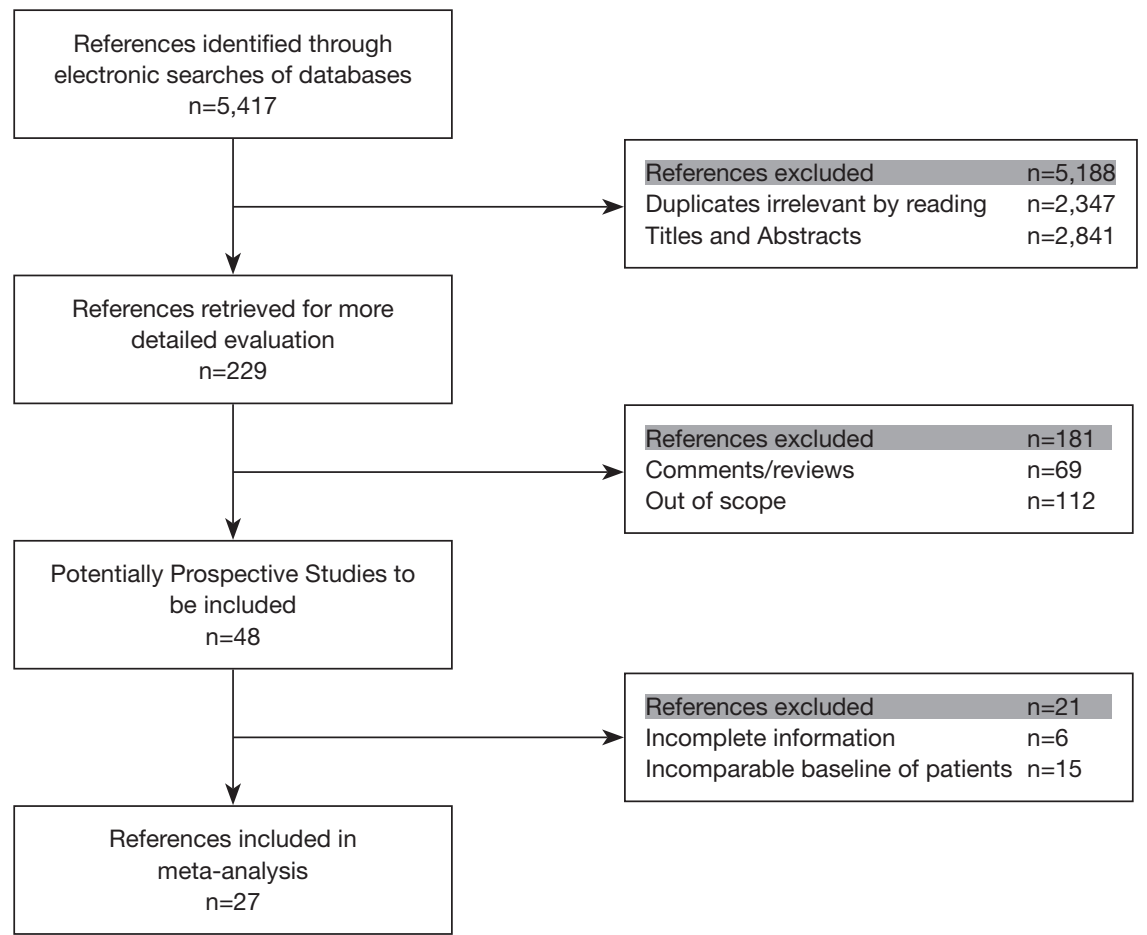

Figure 1 Identification of studies for inclusion.

The anastomosis could be done mainly in four different ways: (I) a running suture for the membranous part and single stitches for the cartilage (knots are tied outwards); (II) double continuous suture; (III) a single continuous suture for the whole anastomosis; (IV) fully interrupted sutures.

No difference in definitive outcome is observed by the different suture techniques.

Before proceeding to the bronchial anastomosis, a pathological frozen section confirmation of neoplastic cells absence in the proximal margin is required. At the end of the suture, the air leakage check is performed by submerging the lung in saline solution and re-ventilating it. If necessary, intra-operative fiberoptic bronchoscopy could be performed to examine the anastomosis directly. Usually, the bronchial anastomosis and the vascular structures are separated by vital pedicled pleura and pericardial fat, to prevent possible fistula. Additionally, a sleeve resection of more than 1 lobe with atypical bronchial resection and reconstruction was terminated as an extended SL by Okada et al. The extended procedure could be more complicated than the standard SL, and was classified as Okada type A-D based on the extent of resection and location of anastomose. Generally, lymph-node resection, according to the international guidelines (14) is performed before completing the bronchial anastomosis.

\section{Statistical analysis}

Statistical analysis was performed using the software package RevMan 5.1.0. Odds ratio (OR) or mean difference with $95 \%$ confidence intervals (95\% CI) were calculated for dichotomous outcomes and continuous outcomes respectively. A random-effects and fixed-effect models were done using 'intention-to-treat' analysis. If the results were not different between the two models, the randomeffects model was reported, as this model was used for indirect comparisons. If results differed between the two models, both results were reported. Heterogeneity was explored by $\chi^{2}$ and $\mathrm{I}^{2} . \mathrm{I}^{2}<25 \%$ and $\mathrm{I}^{2}>50 \%$ reflect small and large inconsistency, respectively. If $\mathrm{P}>0.10$, these studies were deemed to exhibit homogeneity and a fixedeffect analysis model was used. When $\mathrm{P}<0.10$ and $\mathrm{I}^{2}<50 \%$, these studies were considered to exhibit heterogeneity, but the heterogeneity could be accepted, and a fixed-effect analysis model was used too. When $\mathrm{P}<0.10$ and $\mathrm{I}^{2}>50 \%$, the heterogeneity was too high to be accepted, and a randomeffect analysis model was used. A P value of $<0.05$ was considered significant. 


\section{Subgroup analysis}

A subgroup analysis was performed by including patients with pathological N0, N1 and N2 diseases.

\section{Publication bias}

We used funnel plots model to check for bias. Asymmetry in the funnel plot of trial size against treatment effect was used to assess the risk of bias.

\section{Results}

\section{Description of studies}

After screening (Figure 1), 27 studies were included. Of 14,194 patients in these 27 studies, 4,145 were allocated to the SL group, whereas 10,049 to the PN group. Patient characteristics and evaluation index are shown in Table 1. Selected articles were ranked $7-8$ on a quality-indicator scale by Newcastle-Ottawa scale (NOS). Results of the meta-analysis are summarized in Table 2 .

Operative mortality (operating room and the perioperative period after surgery)

The meta-analysis $\{13$ trials reported this data include 22 [839] and 130 [2,064] patients in SL and PN\} showed operative mortality in the $\mathrm{PN}$ group was significantly higher than in the SL group (6.30\% vs. 2.62\%) (OR: 0.40; 95\% CI: $0.25-0.63 ; \mathrm{P}<0.0001)$, with no evidence of significant heterogeneity.

\section{0-day mortality}

Twelve trials reported data finclude $89[3,195]$ and $463[7,890]$ patients in SL and PN\} on 30-day mortality and showed that there was statistical difference between SL and PN relative to 30 -day mortality (2.78\% vs. $5.86 \%$ ) (OR: 0.55 ; 95\% CI: $0.32-0.96 ; \mathrm{P}=0.04)$, with specific heterogeneity.

\section{Complications}

Concerning to perioperative complications, the metaanalysis (15 trials reported this data) showed that there was no statistically significant difference between the SL and the PN groups (OR: 1.07; 95\% CI: 0.87-1.31; $\mathrm{P}=0.55$ ), with specific heterogeneity.

\section{Local recurrence rates}

Fifteen trials reported data on local recurrence. There was no statistically significant difference between the SL and the PN groups (OR: 1.09; 95\% CI: 0.72-1.64; P=0.69), with specific heterogeneity.

\section{Distant recurrence rates}

Nine trials reported data on distant recurrence rates and demonstrated a higher risk of distant recurrence with PN compared to SL (OR: 0.61; 95\% CI: 0.45-0.82; $\mathrm{P}=0.001$ ), with no evidence of significant heterogeneity (Figure 2).

\section{Overall survival rates}

One-year survival rates: the meta-analysis ( 8 trials reported this data) showed that the group of SL experienced a significantly higher survival rate compared to PN (OR: 1.53; $95 \%$ CI: $1.31-1.80 ; \mathrm{P}<0.00001)$, with no evidence of significant heterogeneity.

Three-year survival rates: the meta-analysis (11 trials reported this data) showed that survival in PN group was significantly lower compared to SL (OR: 1.78; 95\% CI: 1.47-2.17; $\mathrm{P}<0.00001$ ), with specific heterogeneity (Figure 3).

Five-year survival rates: the meta-analysis (20 trials reported this data) showed that survival in PN group as significantly lower compared to SL one (OR: 1.96; 95\% CI: 1.70-2.27; $\mathrm{P}<0.00001$ ), with specific heterogeneity (Figure 4).

\section{Comparison of overall survival rates between the two groups in NO and N1, N2 (pathological) patients}

When survival was evaluated according to the lymph node involvement and the type of resection, the results of 5 trials demonstrated improved 5-year overall survival rates for patients with N0-N1 disease who underwent SL as compared to PN (OR: 2.14; 95\% CI: 1.66-2.78; $\mathrm{P}<0.00001$ ), with specific heterogeneity (Figure 5). In patients with N2 disease, there was no statistically significant difference in 3or 5 -year overall survival concerning the type of resection (OR: 1.12; 95\% CI: 0.47-2.68; P=0.79; OR: 1.27; 95\% CI: $0.65-2.45 ; \mathrm{P}=0.48)$, with specific heterogeneity.

\section{Publication bias and sensitivity analysis}

When no significant findings remain unpublished, the publication bias may exist, thus artificially inflating the 
Table 1 Characteristics of included trials

\begin{tabular}{|c|c|c|c|c|c|c|c|c|c|c|}
\hline Author, years & Country & Type of study & Treatment & $\begin{array}{c}\text { No. of } \\
\text { patients }\end{array}$ & Gender (M/F) & $\begin{array}{l}\text { Mean age, } \\
\text { years }\end{array}$ & \multicolumn{4}{|c|}{ Stage } \\
\hline \multirow{2}{*}{$\begin{array}{l}\text { Gaissert (15), } \\
1996\end{array}$} & \multirow[t]{2}{*}{ USA } & \multirow[t]{2}{*}{ Cohort } & SL & 72 & $56 / 16$ & - & 29 & 31 & 12 & 0 \\
\hline & & & PN & 56 & $42 / 14$ & - & 9 & 25 & 21 & 1 \\
\hline $\begin{array}{l}\text { Yoshino (10), } \\
1997\end{array}$ & Japan & $\begin{array}{l}\text { Retrospective } \\
\text { case-control }\end{array}$ & SL & 29 & $26 / 3$ & $60.6 \pm 8.7^{\dagger}$ & 9 & 12 & 8 & 0 \\
\hline \multirow{2}{*}{$\begin{array}{l}\text { Suen (11), } \\
1999\end{array}$} & \multirow[t]{2}{*}{ USA } & \multirow[t]{2}{*}{ Cohort } & SL & 58 & $41 / 17$ & $63.7^{\ddagger}$ & 18 & 28 & 12 & 0 \\
\hline & & & PN & 142 & $81 / 61$ & $66.5^{\ddagger}$ & 37 & 46 & 58 & 1 \\
\hline \multirow{2}{*}{$\begin{array}{l}\text { Okada (13), } \\
2000\end{array}$} & \multirow[t]{2}{*}{ Japan } & \multirow{2}{*}{$\begin{array}{l}\text { Retrospective } \\
\text { case-control }\end{array}$} & SL & 60 & $52 / 8$ & $60.9 \pm 9.5^{\dagger}$ & - & - & - & - \\
\hline & & & PN & 60 & $53 / 7$ & $60.6 \pm 9.0^{\dagger}$ & - & - & - & - \\
\hline \multirow{2}{*}{$\begin{array}{l}\text { Ghiribelli (17), } \\
2002\end{array}$} & \multirow[t]{2}{*}{ Italy } & \multirow[t]{2}{*}{ Cohort } & SL & 38 & $36 / 2$ & $65^{\ddagger}$ & 16 & 10 & 12 & 0 \\
\hline & & & PN & 127 & $102 / 25$ & $62.4^{\ddagger}$ & 29 & 43 & 55 & 0 \\
\hline Deslauriers & Canada & Cohort & SL & 184 & $152 / 32$ & $60.0 \pm 10.0^{\dagger}$ & 82 & 72 & 30 & 0 \\
\hline (8), 2004 & & & PN & 1,046 & $827 / 219$ & $60.7 \pm 9.4^{\dagger}$ & 164 & 361 & 466 & 55 \\
\hline Kim (18), & Korea & Retrospective & SL & 49 & $44 / 5$ & $58.7 \pm 7.6^{\dagger}$ & 14 & 20 & 15 & 0 \\
\hline 2005 & & case-control & PN & 49 & $46 / 3$ & $58.1 \pm 8.2^{\dagger}$ & 24 & 13 & 11 & 1 \\
\hline Ludwig (19), & Germany & Cohort & SL & 116 & - & $62^{\ddagger}$ & 31 & 41 & 44 & 0 \\
\hline 2005 & & & PN & 194 & - & $59^{\ddagger}$ & 32 & 52 & 110 & 0 \\
\hline 2006 & & & PN & 110 & $92 / 18$ & $59.3 \pm 9.6^{\dagger}$ & 24 & 14 & 70 & 2 \\
\hline Jiménez (22), & Spain & Cohort & SL & 35 & $34 / 1$ & $62^{\ddagger}$ & - & - & - & - \\
\hline 2006 & & & PN & 220 & $205 / 5$ & $62^{\ddagger}$ & - & - & - & - \\
\hline Balduyck & Belgium & Cohort & SL & 10 & - & $65.3^{\ddagger}$ & 2 & 1 & 7 & 0 \\
\hline (23), 2008 & & & PN & 20 & - & $63.3^{\ddagger}$ & 3 & 9 & 8 & 0 \\
\hline Melloul (24), & Switzerland & Cohort & SL & 69 & - & - & - & - & - & - \\
\hline 2008 & & & PN & 78 & - & - & - & - & - & - \\
\hline Parissis (25), & Ireland & Cohort & SL & 79 & $54 / 25$ & $60.4^{\ddagger}$ & - & - & - & - \\
\hline 2009 & & & PN & 129 & $91 / 38$ & $62.5^{\ddagger}$ & - & - & - & - \\
\hline Hanagiri (26), & Japan & Cohort & SL & 24 & $18 / 6$ & $65.1^{\ddagger}$ & 5 & 8 & 10 & 1 \\
\hline 2010 & & & PN & 72 & $61 / 11$ & $64.7^{\ddagger}$ & 5 & 13 & 50 & 4 \\
\hline
\end{tabular}

Table 1 (continued) 
Table 1 (continued)

\begin{tabular}{|c|c|c|c|c|c|c|c|c|c|c|}
\hline Author, years & Country & Type of study & Treatment & $\begin{array}{c}\text { No. of } \\
\text { patients }\end{array}$ & Gender (M/F) & $\begin{array}{l}\text { Mean age, } \\
\text { years }\end{array}$ & \multicolumn{4}{|c|}{ Stage } \\
\hline \multirow{2}{*}{$\begin{array}{l}\text { Park (27), } \\
2010\end{array}$} & \multirow[t]{2}{*}{ Korea } & \multirow{2}{*}{$\begin{array}{l}\text { Retrospective } \\
\text { case-control }\end{array}$} & SL & 105 & $99 / 6$ & $61.25 \pm 8.89^{\dagger}$ & 44 & 32 & 26 & 3 \\
\hline & & & PN & 105 & $98 / 7$ & $62.24 \pm 8.42^{\dagger}$ & 43 & 36 & 24 & 2 \\
\hline $\begin{array}{l}\text { Bölükbas } \\
(28), 2011\end{array}$ & Germany & Cohort & SL & 31 & $25 / 6$ & $73.6^{\ddagger}$ & 5 & 17 & 8 & 1 \\
\hline \multirow{2}{*}{$\begin{array}{l}\text { Gómez-Caro } \\
(29), 2011\end{array}$} & \multirow[t]{2}{*}{ Spain } & \multirow[t]{2}{*}{ Cohort } & SL & 55 & $51 / 4$ & $63.5 \pm 10.2^{\dagger}$ & 19 & 27 & 9 & 0 \\
\hline & & & PN & 21 & $18 / 3$ & $62.4 \pm 8.2^{\dagger}$ & 3 & 14 & 4 & 0 \\
\hline \multirow{2}{*}{$\begin{array}{l}\text { Lee (30), } \\
2011\end{array}$} & \multirow[t]{2}{*}{ Korea } & \multirow[t]{2}{*}{ Cohort } & SL & 19 & $15 / 4$ & $62.1 \pm 8.9^{\dagger}$ & 5 & 8 & 6 & 0 \\
\hline & & & PN & 20 & $16 / 4$ & $64.3 \pm 8.8^{\dagger}$ & 8 & 5 & 7 & 0 \\
\hline \multirow{2}{*}{$\begin{array}{l}\text { Cusumano } \\
(31), 2014\end{array}$} & \multirow[t]{2}{*}{ Italy } & \multirow[t]{2}{*}{ Cohort } & SL & 51 & $40 / 11$ & $63.0 \pm 8.2^{\dagger}$ & 0 & 7 & 44 & 0 \\
\hline & & & PN & 68 & $54 / 14$ & $59.7 \pm 10.7^{\dagger}$ & 0 & 3 & 65 & 0 \\
\hline \multirow{2}{*}{$\begin{array}{l}\text { Andersson } \\
\text { (32), } 2015\end{array}$} & \multirow[t]{2}{*}{ Finland } & \multirow[t]{2}{*}{ Cohort } & SL & 40 & $29 / 11$ & 61.5 & 8 & 19 & 13 & 0 \\
\hline & & & PN & 67 & $49 / 18$ & 60 & 16 & 26 & 25 & 0 \\
\hline \multirow[t]{2}{*}{ Ma (33), 2016} & \multirow[t]{2}{*}{ China } & \multirow[t]{2}{*}{ Cohort } & SL & 58 & $50 / 8$ & $58.5 \pm 10.3^{\dagger}$ & 0 & 30 & 28 & 0 \\
\hline & & & PN & 42 & $40 / 2$ & $57.8 \pm 7.9^{\dagger}$ & 0 & 18 & 40 & 0 \\
\hline \multirow{2}{*}{$\begin{array}{l}\text { Pagès (34), } \\
2016\end{array}$} & France & Cohort & SL & 941 & $716 / 225$ & $60.9 \pm 12.6^{\dagger}$ & 190 & 408 & 169 & 32 \\
\hline & & & PN & 5,318 & $4,216 / 1,102$ & $61.9 \pm 10.2^{\dagger}$ & 347 & 1,813 & 1,482 & 690 \\
\hline
\end{tabular}

${ }^{\dagger}$, mean \pm standard deviation; ${ }^{\ddagger}$, mean. SL, sleeve lobectomy; PN, pneumonectomy; M, male; F, female.

apparent magnitude of an effect. Survival and local recurrence rates following SL or PN for the treatment of NSCLC were calculated by the fixed-effect model and random-effect model, respectively. The two results are similar, and the combination of the two results makes them more reliable.

Funnel plots of the study results are shown in Figure 6. The funnel plots on 5-year overall survival rates following SL or PN for the treatment of NSCLC showed symmetry, which suggested there was no publication bias.

\section{Discussion}

Sleeve resection for lung cancer can prevent more lung parenchyma sacrifice, limiting the physiologic effects of PN. Many studies published over the past 20 years show similar or better results for parenchymal sparing resections compared to PN. Both Deslauriers (8) and Okada (13) reported a better prognosis after SL in patients with stages I and II NSCLC. Takeda (21) did not show any difference in 5 -year survival for stage I and II patients after SL or PN, but the overall 5-year survival in the SL group was better than in the PN group (54\% vs. 33\%). In this meta-analysis 1-, 3-, 5 -year survival rates were $18.26 \%, 10.95 \%, 7.34 \%$ in the PN group and $38.00 \%, 27.80 \%, 25.77 \%$ in the SL group respectively, which is in line with the data of the literature.

From the oncological point of view, each patient must have an en-bloc complete resection of the tumor to ensure that the margin of resection is negative. Induction therapy has shown to improve local and systemic control in locally-advanced NSCLC (36-38). However, induction therapy may cause fibrosis and treatment-related changes, which make dissection of lobar bronchus or artery and 
Table 2 Summary of the results between SL and PN in the management of NSCLC

\begin{tabular}{|c|c|c|c|c|c|c|}
\hline Variables & No. of studies furnishing data & \multicolumn{2}{|c|}{ Results, \% } & OR $(95 \% \mathrm{Cl})$ & $P$ value & $1^{2}, \%$ \\
\hline Operative mortality & $13(8,10,11,17-19,23-28,30)$ & 2.62 & 6.30 & $0.40(0.25-0.63)$ & $<0.0001$ & 0 \\
\hline 30-day mortality & $12(14-16,20-22,29,32-36)$ & 2.78 & 5.86 & $0.55(0.32-0.96)$ & 0.04 & 55 \\
\hline Local recurrence & $15(8,10,13,16,17,19,21,25,27,28,30-34)$ & 15.65 & 22.81 & $1.09(0.72-1.64)$ & 0.69 & 50 \\
\hline Complication & $15(10,13,14,16-21,24,28,29,31-33)$ & 29.39 & 30.58 & $1.07(0.87-1.31)$ & 0.55 & 27 \\
\hline \multicolumn{7}{|l|}{ Overall survival } \\
\hline 1-year & $8(11,14,15,20,21,28,29,35)$ & 38.00 & 18.26 & $1.53(1.31-1.80)$ & $<0.00001$ & 4 \\
\hline 3-year & $11(11,13,17,20,21,27-30,32,35)$ & 27.80 & 10.95 & $1.78(1.47-2.17)$ & $<0.00001$ & 30 \\
\hline 3-year (N2 patients) & $3(13,17,22)$ & 29.78 & 19.51 & $1.12(0.47-2.68)$ & 0.79 & 35 \\
\hline 5-year (N2 patients) & $3(8,13,18)$ & 19.77 & 18.69 & $1.27(0.65-2.45)$ & 0.48 & 44 \\
\hline $\begin{array}{l}\text { 5-year (N0 and N1 } \\
\text { patients) }\end{array}$ & $5(8,13,17,18,22)$ & 57.77 & 37.29 & $2.14(1.66-2.78)$ & $<0.00001$ & 13 \\
\hline
\end{tabular}

SL, sleeve lobectomy; PN, pneumonectomy; NSCLC, non-small cell lung cancer; OR, odds ratio; 95\% Cl, 95\% confidence interval.

\begin{tabular}{|c|c|c|c|c|c|c|c|c|}
\hline Study or Subgroup & $\begin{array}{r}\text { SL } \\
\text { Events }\end{array}$ & Total & $\begin{array}{r}\text { PN } \\
\text { Events }\end{array}$ & Total & Weight & $\begin{array}{c}\text { Odds Ratio } \\
\text { M-H, Fixed, } 95 \% \text { Cl }\end{array}$ & $\begin{array}{r}\text { Odds } \\
\text { M-H, Fixe }\end{array}$ & $\begin{array}{l}\text { Ratio } \\
\text { ed, } 95 \% \mathrm{Cl}\end{array}$ \\
\hline Andersson 2015 & 13 & 40 & 22 & 67 & $10.5 \%$ & $0.98[0.43,2.27]$ & & - \\
\hline Bolukbas 2011 & 6 & 31 & 7 & 29 & $5.5 \%$ & $0.75[0.22,2.59]$ & & \\
\hline Cusumano 2014 & 9 & 51 & 24 & 68 & $16.0 \%$ & $0.39[0.16,0.94]$ & & \\
\hline Lee 2011 & 3 & 19 & 7 & 20 & $5.4 \%$ & $0.35[0.07,1.62]$ & & \\
\hline Ma 2016 & 13 & 58 & 8 & 42 & $6.8 \%$ & $1.23[0.46,3.29]$ & & \\
\hline Maurizi 2013 & 6 & 39 & 11 & 39 & $8.8 \%$ & $0.46[0.15,1.41]$ & & \\
\hline Park 2010 & 12 & 105 & 23 & 105 & $19.2 \%$ & $0.46[0.22,0.98]$ & & \\
\hline Takeda 2006 & 18 & 62 & 47 & 110 & $22.7 \%$ & $0.55[0.28,1.07]$ & $\rightarrow$ & \\
\hline Yoshino 1997 & 6 & 29 & 7 & 29 & $5.2 \%$ & $0.82[0.24,2.83]$ & & \\
\hline Total $(95 \% \mathrm{Cl})$ & & 434 & & 509 & $100.0 \%$ & $0.61[0.45,0.82]$ & 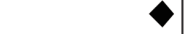 & \\
\hline Total events & 86 & & 156 & & & & & \\
\hline $\begin{array}{l}\text { Heterogeneity: } \mathrm{Chi}^{2}= \\
\text { Test for overall effect }\end{array}$ & $\begin{array}{l}87, \mathrm{df}=8 \\
=3.20(\mathrm{~F}\end{array}$ & $\begin{array}{l}(P=0 \\
=0.0 C\end{array}$ & $\begin{array}{l}66) ; I^{2}= \\
\text { 1) }\end{array}$ & & & & $\begin{array}{ll}0.01 & 0.1\end{array}$ & $\begin{array}{lll}1 & 10 & 100\end{array}$ \\
\hline
\end{tabular}

Figure 2 Distant recurrence rates of SL vs. PN. SL, sleeve lobectomy; PN, pneumonectomy; OR, odds ratio; 95\% CI, 95\% confidence interval.

reconstructive procedures more complex. Okada (13) described that there was a significant difference among patient classification of nodal disease $\mathrm{N} 0$ or $\mathrm{N} 1$ in favor of SL. Moreover, Deslauriers (8) reported that among patients with N0 disease there was a significant difference in favor SL. Mehran and colleagues (39) demonstrated a significant difference in survival between $\mathrm{N} 1$ and $\mathrm{N} 2$ disease irrespectively to the type of surgical resection performed, but they found no difference between N0 and N1. Van Schil and colleagues (40) showed there was a highly significant difference in survival between patients with $\mathrm{N} 0$ and N1/N2 disease, even if a similar difference in survival was not observed between N1 and N2. Finally, both Okada (13) and Deslauriers (8) showed that there was no significant difference in survival in N2 disease when the type of surgery was taken into account. Mehran and 


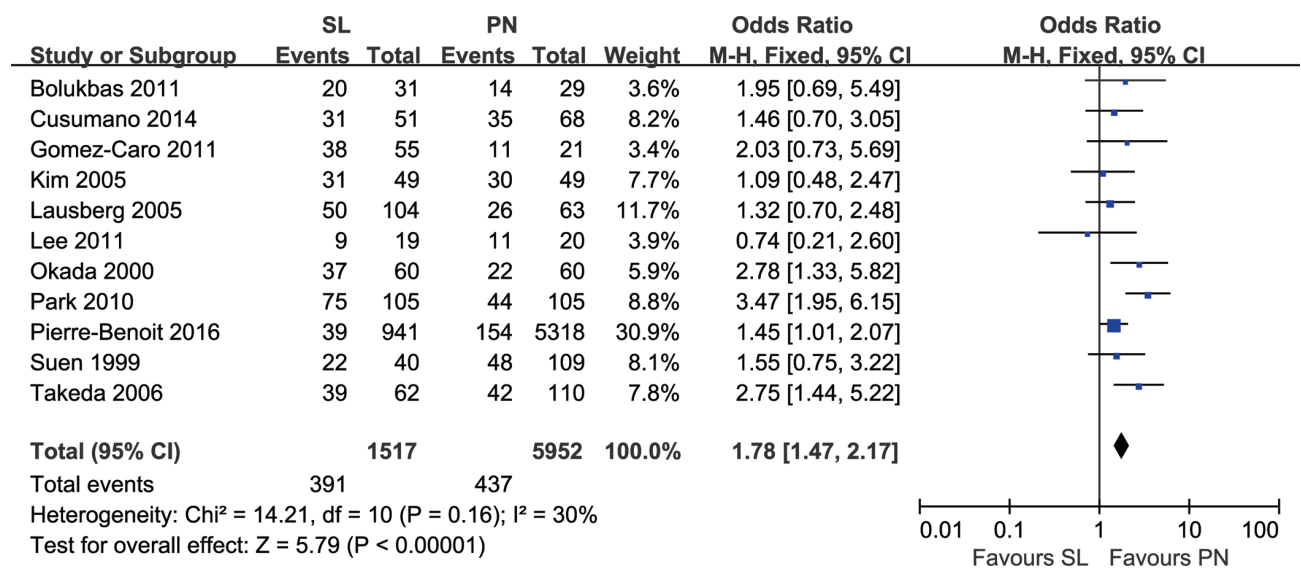

Figure 3 Three-years overall survival rates for SL vs. PN. SL, sleeve lobectomy; PN, pneumonectomy; OR, odds ratio; 95\% CI, 95\% confidence interval.

\begin{tabular}{|c|c|c|c|c|c|c|c|}
\hline Study or Subgroup & $\begin{array}{r}\text { SL } \\
\text { Events }\end{array}$ & Total & $\begin{array}{r}\text { PN } \\
\text { Events }\end{array}$ & Total & Weight & $\begin{array}{l}\text { Odds Ratio } \\
\text { M-H. Fixed. } 95 \% \mathrm{Cl}\end{array}$ & $\begin{array}{c}\text { Odds Ratio } \\
\text { M-H. Fixed. } 95 \% \mathrm{Cl}\end{array}$ \\
\hline Andersson 2015 & 15 & 40 & 28 & 67 & $5.2 \%$ & $0.84[0.37,1.87]$ & $\rightarrow$ \\
\hline Gaissert 1996 & 30 & 72 & 25 & 56 & $6.5 \%$ & $0.89[0.44,1.79]$ & 一 \\
\hline Suen 1999 & 11 & 29 & 28 & 78 & $3.7 \%$ & $1.09[0.45,2.63]$ & $\leftarrow$ \\
\hline Ma 2016 & 37 & 58 & 25 & 42 & $4.2 \%$ & $1.20[0.53,2.71]$ & \\
\hline Lausberg 2005 & 23 & 104 & 12 & 63 & $4.6 \%$ & $1.21[0.55,2.64]$ & - \\
\hline Kim 2005 & 29 & 49 & 26 & 49 & $4.2 \%$ & $1.28[0.58,2.85]$ & - \\
\hline Parissis 2009 & 37 & 79 & 48 & 129 & $7.7 \%$ & $1.49[0.84,2.62]$ & - \\
\hline Cusumano 2014 & 27 & 51 & 29 & 68 & $4.6 \%$ & $1.51[0.73,3.14]$ & - \\
\hline Takeda 2006 & 22 & 62 & 28 & 110 & $5.2 \%$ & $1.61[0.82,3.16]$ & - \\
\hline Ludwig 2005 & 45 & 116 & 52 & 194 & $9.4 \%$ & $1.73[1.06,2.83]$ & $\sigma$ \\
\hline Ghiribelli 2002 & 14 & 36 & 30 & 122 & $3.3 \%$ & $1.95[0.89,4.29]$ & 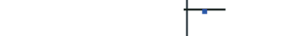 \\
\hline Okada 2000 & 29 & 60 & 17 & 60 & $3.5 \%$ & $2.37[1.11,5.04]$ & $=$ \\
\hline Bagan 2005 & 36 & 63 & 47 & 132 & $5.2 \%$ & $2.41[1.31,4.45]$ & 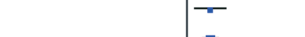 \\
\hline Deslauriers 2004 & 96 & 184 & 324 & 1046 & $18.4 \%$ & $2.43[1.77,3.34]$ & 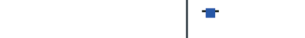 \\
\hline Pierre-Benoit 2016 & 10 & 941 & 20 & 5318 & $2.4 \%$ & $2.85[1.33,6.10]$ & $\longrightarrow$ \\
\hline Jimenez 2006 & 20 & 35 & 70 & 220 & $3.3 \%$ & $2.86[1.38,5.91]$ & 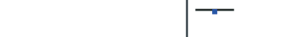 \\
\hline Park 2010 & 61 & 105 & 34 & 105 & $5.7 \%$ & $2.90[1.65,5.09]$ & - \\
\hline Gomez-Caro 2011 & 34 & 55 & 7 & 21 & $1.5 \%$ & $3.24[1.12,9.33]$ & \\
\hline Hanagiri 2010 & 17 & 24 & 24 & 72 & $1.4 \%$ & $4.86[1.77,13.30]$ & \\
\hline Bolukbas 2011 & 18 & 31 & 0 & 29 & $0.1 \%$ & $80.85[4.53,1443.05]$ & \\
\hline Total $(95 \% \mathrm{Cl})$ & & 2194 & & 7981 & $100.0 \%$ & $1.96[1.70,2.27]$ & 1 \\
\hline Total events & 611 & & 874 & & & & \\
\hline \multicolumn{8}{|c|}{ 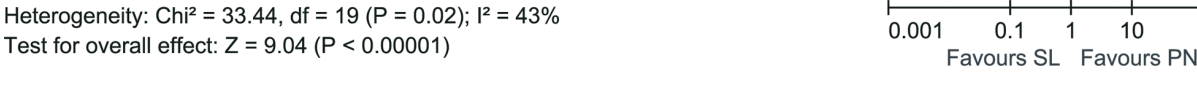 } \\
\hline
\end{tabular}

Figure 4 Five-years overall survival rates for SL vs. PN. SL, sleeve lobectomy; PN, pneumonectomy; OR, odds ratio; 95\% CI, 95\% confidence interval.

colleagues (39) reported that no patients with N2 disease survived longer than 5 years after sleeve resection, and Okada and colleagues (13) reported a $21 \%$-year survival rate after sleeve resection in the same subgroup of patients. In this meta-analysis, a better 5 -year OS was observed after SL in case of N0 and N1 patients, but no difference in 3and 5-year OS was observed in case of N2 disease.

Recent reports have shown that an SL can be performed with a much lower rate of operative mortality (1.2-7.5\%) as compared with PN (4.9-12.0\%) $(8,9,12,19,41)$. SL was a safe procedure with few operative morbidities $(10,15,42,43)$. In a meta-analysis, Ferguson and Lehman (9) reported that the weighted mean operative mortality was $4.1 \%$ after SL and $6.0 \%$ after $\mathrm{PN}(\mathrm{P}=0.3)$. Deslauriers and colleagues (8) described four times greater operative mortality after $\mathrm{PN}$ compared to SL (5.3\% vs. 1.6\%, P=0.036). Recently, SL mortality has been reported ranging between $2 \%$ (37) and $5 \%$ (44). Also, our results confirm this trend and are in line 


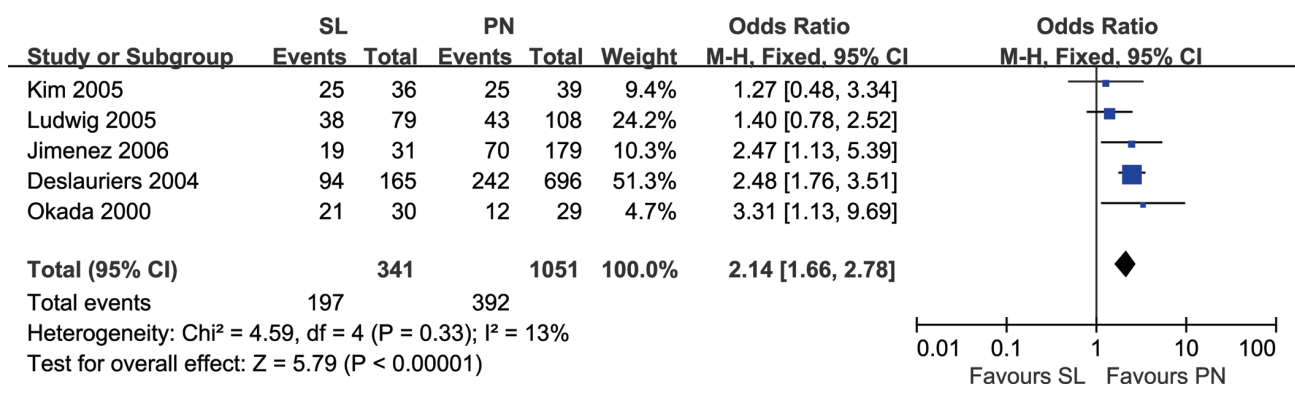

Figure 5 Five-year overall survival-N0 and N1 patients. SL, sleeve lobectomy; PN, pneumonectomy; OR, odds ratio; 95\% CI, 95\% confidence interval.

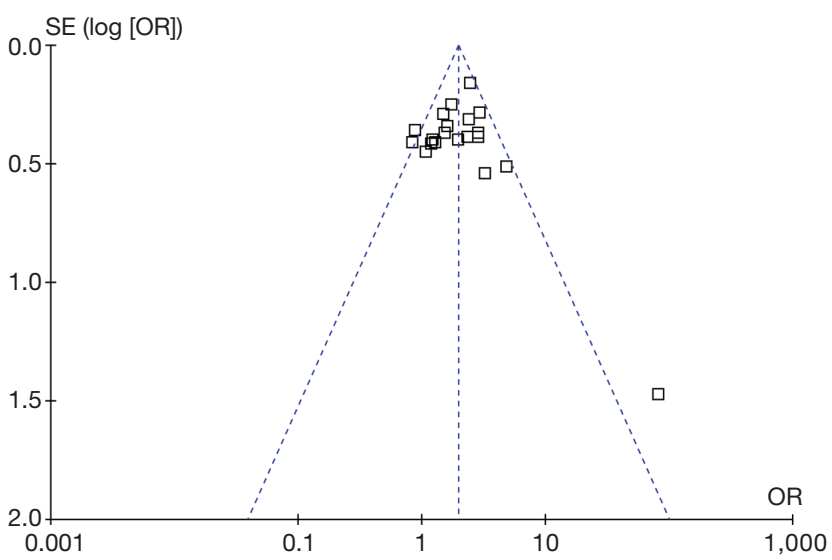

Figure 6 Funnel plot 5 -year overall survival rates. SE, standard error; OR, odds ratio.

with those of the international literature.

Our study showed that there was no statistically significant difference in 30-day mortality. Deslauriers is often quoted as saying that "pneumonectomy is a disease". The main causes of death after PN were: circulation complications [heart failure (45)], acute respiratory failure, acute respiratory distress syndrome (ARDS) (46), arrhythmia and renal disease. Compared with SL, PN can cause sudden and significant hemodynamic changes, hypoxemia, and mediastinal displacement. PN can also predispose to arrhythmia. Right ventricular overload and dysfunction can occur as a result of pulmonary hypertension and subtle fluid overload is often poorly tolerated. Postoperative quality of life has been advocated as one of the most reliable indicators that should influence the decision to perform an SL rather than a PN. Several studies indicate that lung parenchyma sparing improves postoperative quality of life because of a greater pulmonary reserve. Gómez-Caro (29) and Melloul (24) reported there was a statistically significant difference favoring SL in terms of postoperative loss of forced expiratory volume in 1 second (FEV1).

On the other hand, SL may have a unique set of complications in its own right. Anastomotic dehiscence may be life-threatening, and often completion $\mathrm{PN}$ is needed. Anastomotic stenosis and consequent obstructive atelectasis are also major complications after SL, while postpneumonectomy empyema and respiratory failure are the most common complications after PN. Our study reports that the rate of postoperative complications was $29.39 \%$ and $30.58 \%$ in the SL and PN group, respectively, without statistically significant difference.

We may be concerned that bronchoplasty for malignant tumors may increase the potential for local recurrence. According to Tedder and colleagues (44), the local recurrence rate was $13 \%$ after SL. Other series reported an estimated $20 \%$ to $23 \%$ rate of local recurrence (39). In this study, the weighted $15.6 \%$ mean local recurrence rate after SL and $22.8 \%$ after $\mathrm{PN}(\mathrm{P}=0.69)$. The significant lower recurrence rate after SL $(19.81 \%$ vs. $30.64 \%$ after $\mathrm{PN}$, $\mathrm{P}=0.001$ ) might also explain why the SL group experienced a better overall survival. The lower distant recurrence rate is an essential explanation in the survival advantage of SL, and the survival also may be influenced by the lesser physiological insult of SL. Alterations in immune response following the physiologic changes with $\mathrm{PN}$ have been suggested to play a role in distant recurrence (47).

\section{Limitations of the study}

The conclusions of our study may be limited by several factors. First, the studies analyzed have a very long period. This can lead to false positive or false negative results (risk of random errors). Second, not all data comes from RCTs. Therefore, the overall level of clinical evidence was 
relatively low.

There was a concern for publication bias in the studies included. Those surgeons with less than optimal experiences or outcomes inferior to a thoracotomy would likely be less than enthusiastic about publishing their data if they were accepted for publication at all. Moreover, there are many subtle differences in technique between surgeons. Also, funnel plots can show the cause of asymmetry rather than publication bias. As a result, our merger may overestimate the real effect. Due to these data limitations, our meta-analysis was unable to analyze the quality of life score and stratified analysis of other possible confounding factors. If the method is to be more effective, then larger samples and randomized controlled studies with longer follow-up are required.

Finally, we failed to perform a separated analysis according to the tumor's histology because of the very limited data available. Darling's IIIA NSCLC study and Deslauriers excellent results indicated that if the surgery is performed the surgery it has some clinical impact.

\section{Conclusions}

Our study demonstrates improved survival following SL vs. $\mathrm{PN}$ in patients with NSCLC. This effect was irrespective of lymph node involvement when the disease was N0-N1. The presence of mediastinal lymph node metastases (N2) portended worse survival, that was irrespective of the type of surgery. SL is a useful alternative to $\mathrm{PN}$ for operable cases of lung cancers and should be taken into account. Further RCTs are warranted to clarify the exact value of SL and PN for NSCLC to get a higher level of evidence.

\section{Acknowledgments}

Funding: This project was supported by grants from Department of Liaoning Science and Technology (1564992449013), titled with The Construction of Liaoning Cancer Research Center (Lung Cancer); and titled with Precise diagnosis and treatment and optimization of clinical pathway for malignant tumor based on molecular markersthe research of precise treatment and optimization of clinical pathway for lung cancer, and 2019 Grant to the Construction of Provincial Key Specialty;and Research grant to introduced talents of Liaoning Cancer Hospital.

\section{Footnote}

Conflicts of Interest: The authors have no conflicts of interest to declare.

Ethical Statement: The authors are accountable for all aspects of the work in ensuring that questions related to the accuracy or integrity of any part of the work are appropriately investigated and resolved. The study was approved by institutional ethics committee board of Shenyang Chest Hospital and Cancer Hospital of China Medical University/Liaoning Cancer Hospital (ethical number KYXM-2018-001-01 and 20171125).

\section{References}

1. Thomas CP. Conservative resection of the bronchial tree. J R Coll Surg Edinb 1956;1:169-86.

2. Allison PR. Course of thoracic surgery in Groningen. Ann R Coll Surg 1954;25:20-2.

3. D'Abreu AL, Machale SJ. Bronchial "adenoma" treated by local resection and reconstruction of the left main bronchus. Br J Surg 1952;39:355-7.

4. Gebauer PW. Bronchial resection and anastomosis. J Thorac Surg 1953;26:241-60.

5. Paulson DL, Shaw RR. Preservation of lung tissue by means of bronchoplastic procedures. Am J Surg 1955;89:347-55.

6. Jensik RJ, Faber LP, Milloy FJ, et al. Sleeve lobectomy for carcinoma. A ten-year experience. J Thorac Cardiovasc Surg 1972;64:400-12.

7. Faber LP, Jensik RJ, Kittle CF. Results of sleeve lobectomy for bronchogenic carcinoma in 101 patients. Ann Thorac Surg 1984;37:279-85.

8. Deslauriers J, Grégoire J, Jacques LF, et al. Sleeve lobectomy versus pneumonectomy for lung cancer: a comparative analysis of survival and sites or recurrences. Ann Thorac Surg 2004;77:1152-6; discussion 1156.

9. Ferguson MK, Lehman AG. Sleeve lobectomy or pneumonectomy: optimal management strategy using decision analysis techniques. Ann Thorac Surg 2003;76:1782-8.

10. Yoshino I, Yokoyama H, Yano T, et al. Comparison of the surgical results of lobectomy with bronchoplasty and pneumonectomy for lung cancer. J Surg Oncol 1997;64:32-5.

11. Suen HC, Meyers BF, Guthrie T, et al. Favorable results after sleeve lobectomy or bronchoplasty for bronchial malignancies. Ann Thorac Surg 1999;67:1557-62.

12. Lausberg HF, Graeter TP, Wendler O, et al. Bronchial and bronchovascular sleeve resection for treatment of central 
lung tumors. Ann Thorac Surg 2000;70:367-71; discussion 371-2.

13. Okada M, Yamagishi H, Satake S, et al. Survival related to lymph node involvement in lung cancer after sleeve lobectomy compared with pneumonectomy. J Thorac Cardiovasc Surg 2000;119:814-9.

14. Maurizi G, D'Andrilli A, Anile M, et al. Sleeve lobectomy compared with pneumonectomy after induction therapy for non-small-cell lung cancer. J Thorac Oncol 2013;8:637-43.

15. Gaissert HA, Mathisen DJ, Moncure AC, et al. Survival and function after sleeve lobectomy for lung cancer. J Thorac Cardiovasc Surg 1996;111:948-53.

16. Martin-Ucar AE, Chaudhuri N, Edwards JG, et al. Can pneumonectomy for non-small cell lung cancer be avoided? An audit of parenchymal sparing lung surgery. Eur J Cardiothorac Surg 2002;21:601-5.

17. Ghiribelli C, Voltolini L, Luzzi L, et al. Survival after bronchoplastic lobectomy for non small cell lung cancer compared with pneumonectomy according to nodal status. J Cardiovasc Surg (Torino) 2002;43:103-8.

18. Kim YT, Kang CH, Sung SW, et al. Local control of disease related to lymph node involvement in non-small cell lung cancer after sleeve lobectomy compared with pneumonectomy. Ann Thorac Surg 2005;79:1153-61; discussion 1153-61.

19. Ludwig C, Stoelben E, Olschewski M, et al. Comparison of morbidity, 30-day mortality, and long-term survival after pneumonectomy and sleeve lobectomy for non-small cell lung carcinoma. Ann Thorac Surg 2005;79:968-73.

20. Bagan P, Berna P, Pereira JC, et al. Sleeve lobectomy versus pneumonectomy: tumor characteristics and comparative analysis of feasibility and results. Ann Thorac Surg 2005;80:2046-50.

21. Takeda S, Maeda H, Koma M, et al. Comparison of surgical results after pneumonectomy and sleeve lobectomy for non-small cell lung cancer: trends over time and 20-year institutional experience. Eur J Cardiothorac Surg 2006;29:276-80.

22. Jiménez MF, Varela G, Novoa N, et al. Sleeve lobectomy compared to pneumonectomy for the treatment of N0N1 non-small cell lung cancer. Arch Bronconeumol 2006;42:160-4.

23. Balduyck B, Hendriks J, Lauwers P, et al. Quality of life after lung cancer surgery: a prospective pilot study comparing bronchial sleeve lobectomy with pneumonectomy. J Thorac Oncol 2008;3:604-8.

24. Melloul E, Egger B, Krueger T, et al. Mortality, complications and loss of pulmonary function after pneumonectomy vs. sleeve lobectomy in patients younger and older than 70 years. Interact Cardiovasc Thorac Surg 2008;7:986-9.

25. Parissis H, Leotsinidis M, Hughes A, et al. Comparative analysis and outcomes of sleeve resection versus pneumonectomy. Asian Cardiovasc Thorac Ann 2009;17:175-82.

26. Hanagiri T, Baba T, Ichiki Y, et al. Sleeve lobectomy for patients with non-small cell lung cancer. Int J Surg 2010;8:39-43.

27. Park JS, Yang HC, Kim HK, et al. Sleeve lobectomy as an alternative procedure to pneumonectomy for non-small cell lung cancer. J Thorac Oncol 2010;5:517-20.

28. Bölükbas S, Eberlein MH, Schirren J. Pneumonectomy vs. sleeve resection for non-small cell lung carcinoma in the elderly: analysis of short-term and long-term results. Thorac Cardiovasc Surg 2011;59:142-7.

29. Gómez-Caro A, Garcia S, Reguart N, et al. Determining the appropriate sleeve lobectomy versus pneumonectomy ratio in central non-small cell lung cancer patients: an audit of an aggressive policy of pneumonectomy avoidance. Eur J Cardiothorac Surg 2011;39:352-9.

30. Lee HK, Lee HS, Kim KI, et al. Outcomes of sleeve lobectomy versus pneumonectomy for lung cancer. Korean J Thorac Cardiovasc Surg 2011;44:413-7.

31. Cusumano G, Marra A, Lococo F, et al. Is sleeve lobectomy comparable in terms of short- and long-term results with pneumonectomy after induction therapy? A multicenter analysis. Ann Thorac Surg 2014;98:975-83.

32. Andersson SE, Rauma VH, Sihvo EI, et al. Bronchial sleeve resection or pneumonectomy for non-small cell lung cancer: a propensity-matched analysis of long-term results, survival and quality of life. J Thorac Dis 2015;7:1742-8.

33. Ma QL, Guo YQ, Shi B, et al. For non-small cell lung cancer with T3 (central) disease, sleeve lobectomy or pneumonectomy? J Thorac Dis 2016;8:1227-33.

34. Pagès PB, Mordant $\mathrm{P}$, Renaud $\mathrm{S}$, et al. Sleeve lobectomy may provide better outcomes than pneumonectomy for non-small cell lung cancer. A decade in a nationwide study. J Thorac Cardiovasc Surg 2017;153:184-95.e3.

35. Abdelsattar ZM, Shen KR, Yendamuri S, et al. Outcomes after sleeve lung resections versus pneumonectomy in the United States. Ann Thorac Surg 2017;104:1656-64.

36. Albain KS, Swann RS, Rusch VW, et al. Radiotherapy plus chemotherapy with or without surgical resection for stage III non-small-cell lung cancer: a phase III randomised controlled trial. Lancet 2009;374:379-86.

37. Pisters KM, Vallières E, Crowley JJ, et al. Surgery with or 
without preoperative paclitaxel and carboplatin in earlystage non-small-cell lung cancer: Southwest Oncology Group Trial S9900, an intergroup, randomized, phase III trial. J Clin Oncol 2010;28:1843-9.

38. White A, Kucukak S, Bueno R, et al. Pneumonectomy is safe and effective for non-small cell lung cancer following induction therapy. J Thorac Dis 2017;9:4447-53.

39. Mehran RJ, Deslauriers J, Piraux M, et al. Survival related to nodal status after sleeve resection for lung cancer. J Thorac Cardiovasc Surg 1994;107:576-82; discussion 582-3.

40. Van Schil PE, Brutel de la Rivière A, Knaepen PJ, et al. Second primary lung cancer after bronchial sleeve resection. Treatment and results in eleven patients. J Thorac Cardiovasc Surg 1992;104:1451-5.

41. Stoelben E, Sauerbrei W, Ludwig C, et al. Tumor stage and early mortality for surgical resections in lung cancer. Langenbecks Arch Surg 2003;388:116-21.

42. Icard P, Regnard JF, Guibert L, et al. Survival and prognostic factors in patients undergoing parenchymal saving bronchoplastic operation for primary lung cancer: a series of 110 consecutive cases. Eur J Cardiothorac Surg 1999; 15:426-32.

43. Fadel E, Yildizeli B, Chapelier AR, et al. Sleeve lobectomy for bronchogenic cancers: factors affecting survival. Ann Thorac Surg 2002;74:851-8; discussion 858-9.

44. Tedder M, Anstadt MP, Tedder SD, et al. Current morbidity, mortality, and survival after bronchoplastic procedures for malignancy. Ann Thorac Surg 1992;54:387-91.

45. Schneider L, Farrokhyar F, Schieman C, et al. Pneumonectomy: the burden of death after discharge and predictors of surgical mortality. Ann Thorac Surg 2014;98:1976-81; discussion 1981-2.

46. Stoica RT, Cordoş I, Macri A. Post-pneumonectomy ARDS and ogilvie syndrome - a case report. J Crit Care Med (Targu Mures) 2018;4:34-7.

47. Evans RG, Ndunge OB, Naidu B. A novel two-hit rodent model of postoperative acute lung injury: priming the immune system leads to an exaggerated injury after pneumonectomy. Interact Cardiovasc Thorac Surg 2013;16:844-8.
Cite this article as: $\mathrm{Li} \mathrm{Z,} \mathrm{Chen} \mathrm{W,} \mathrm{Xia} \mathrm{M,} \mathrm{Liu} \mathrm{H,} \mathrm{Liu} \mathrm{Y,} \mathrm{Inci}$ I, Davoli F, Waseda R, Filosso PL, White A. Sleeve lobectomy compared with pneumonectomy for operable centrally located non-small cell lung cancer: a meta-analysis. Transl Lung Cancer Res 2019;8(6):775-786. doi: 10.21037/tlcr.2019.10.11 